\title{
SOME PROBLEMS IN NONLINEAR VOLTERRA INTEGRAL EQUATIONS
}

\author{
BY J. A. NOHEL \\ Communicated by Walter Rudin, March 18, 1962
}

Upper and lower bounds for the norm of solutions of systems of first order differential equations as well as theorems on global existence and boundedness and other useful results have recently been obtained by comparing solutions of the given system with those of a related (single) first order differential equation. This technique, which is essentially due to Conti [5] and Wintner [9], has been extended in scope by Brauer [2;3] and Antosiewicz [1] to obtain many of the results for systems of differential equations referred to above.

In this paper, which will appear in complete form elsewhere, we present a similar technique and using it obtain results for systems of integral equations of the form

$$
x(t)=h(t)+\int_{0}^{t} q(t-\tau) f(\tau, x(\tau)) d \tau \quad(t \geqq 0),
$$

where $h, f$ are given vectors with $n$ components, $q$ is a given $n$ by $n$ matrix, defined on $0 \leqq t<t_{0},|x|<\infty$, for some $t_{0}>0 \quad\left(t_{0}=+\infty\right.$ not excluded). The norm of any vector $x$ with $n$ components is defined as $|x|=\sum_{i=1}^{n}\left|x_{i}\right|$ and the norm of the matrix $q$ as $|q|=\sum_{i, j}\left|q_{i j}\right|$. In order to keep the statements of theorems as simple as possible we restrict ourselves to (1), which is itself sufficiently general to include many applications. It is clear that our results can be modified to include the more general system

$$
x(t)=h(t)+\int_{0}^{t} F(t, \tau, x(\tau)) d \tau \quad(t \geqq 0),
$$

where $F$ is a given vector. We note that some of our assumptions, e.g., in (2) below the continuity of $f$ with respect to $t$, can be relaxed with only minor changes in the proofs.

Essentially the main results are the comparison theorems (Theorems $2.1,2.2$ ) in $\$ 2$. In order to prove these it is necessary to develop for integral equations certain results on local existence without a uniqueness assumption, continuation of solutions, and existence of maximum and minimum solutions for (1) with $n=1$. These are given in $\$ 1$ and are, of course, generalizations of well known theorems for ordinary differential equations. In $\$ 3$ the results and techniques of $\$ 2$ are applied to deduce a general uniqueness result (Theorem 3.1), 
Theorem 3.2 which gives general sufficient conditions for the convergence to a solution of the usual (Picard) successive approximations, and more specific results on global existence and boundedness of solutions of (1) using the comparison theorem. It should be pointed out that our results of $\S \S 2$ and 3 require that the function $w(t, r)$, Theorem 2.1 below, satisfies a monotone condition with respect to $r$. In the corresponding results in ordinary differential equations, except for the analogue of Theorem 3.2, this requirement is not necessary. It appears extremely unlikely that this condition can be dropped in the case of integral equations. Except for this restriction our results contain those mentioned above for ordinary differential equations as very special cases.

1. It will be assumed throughout that

(2) $h, f$ are continuous in $t$ and $(t, x)$ respectively for $0 \leqq t<t_{0}$ and

$$
\begin{gathered}
0 \leqq t<t_{0},|x|<\infty, \\
\text { for any } c, 0<c<t_{0},|q| \in L[0, c] .
\end{gathered}
$$

Lemma 1.1 (Local existence). Let $(2,3)$ be satisfied and let $0<a<t_{0}$. Then there exists a number $\alpha, 0<\alpha \leqq a$, such that (1) has at least one (necessarily continuous) solution on $0 \leqq t \leqq \alpha$. If further for any $A>0$ there exists a constant $K(A)>0$ such that

$$
\left|f\left(t, x_{1}\right)-f\left(t, x_{2}\right)\right| \leqq K(A)\left|x_{1}-x_{2}\right|\left(\left|x_{1}\right|,\left|x_{2}\right| \leqq A\right),
$$

then the solution is unique.

The proof of existence is accomplished without using (4) by showing that the successive approximations $\left\{\phi_{j}\right\}$ (compare with the Caratheodory existence theorem $[4$, p. 43$]$ for ordinary differential equations)

$$
\begin{array}{r}
\phi_{j}(t)=h(t) \\
\phi_{j}(t)=h(t)+\int_{0}^{t-\alpha / j} q(t-\alpha / j-\tau) f\left(\tau, \phi_{j}(\tau)\right) d \tau \\
(\alpha / j \leqq t \leqq \alpha, j=1,2, \cdots)
\end{array}
$$

form a family of continuous vector functions, uniformly bounded and equicontinuous on $0 \leqq t \leqq \alpha$ where $\alpha$ is defined in terms of the quantities

$$
\begin{gathered}
m=\sup _{0 \leqq t \leqq a}|h(t)|, \quad M=\sup _{0 \leqq t \leqq a_{;}|x| \leqq 2 m}|f(t, x)|, \\
P(t)=\int_{0}^{t}|q(\sigma)| d \sigma
\end{gathered}
$$


by the inequality $M P(\alpha) \leqq m$. Since $P(0)=0, P$ is continuous and nondecreasing, such an $\alpha$ exists. Then $\left\{\phi_{j}\right\}$ contains a subsequence converging uniformly on $[0, \alpha]$. This limit function is then shown to satisfy (1). Uniqueness, under the additional assumption (4), follows in the usual way; in fact, with (4) the usual (Picard) approximations converge uniformly to the solution on $[0, \alpha]$. For a further discussion of the convergence of successive approximations and general uniqueness questions we refer to $\$ 3$ below.

The question of continuation of solutions of (1) is answered by means of the following result using the Cauchy criterion and Lemma 1.1.

Lemma 1.2. Let $(2,3)$ be satisfied. Suppose that $\phi$ is a solution of (1) existing on $[0, \beta), \beta \leqq t_{0}$. If $|\phi(t)|$ is bounded on $[0, \beta), \phi\left(\beta^{-}\right)$exists, and if $\beta<t_{0}$, the solution $\phi$ may be continued to the right of $\beta$.

For the remainder of this section we restrict ourselves to the case $n=1$. Analogous to differential equations we have:

DEFINITION. If there exists a continuous solution $\phi_{M}$ of $(1)(n=1)$ on some interval I for $t \geqq 0$, such that every other continuous solution $\phi$ on $I$ satisfies $\phi(t) \leqq \phi_{M}(t), t \in I$, then $\phi_{M}$ is called the maximum solution of (1) on $I$.

The definition of the minimum solution $\phi_{m}$ is similar. The proof of existence of solutions $\phi_{M}, \phi_{m}$ follows closely the one for differential equations [4, pp. 45-57].

Lemma 1.3. Let $(2,3)$ be satisfied. Let $n=1$ and let $\alpha$ be chosen as in Lemma 1.1. Then the maximum solution $\phi_{M}$ and the minimum solution $\phi_{m}$ of $(1)$ exist on $[0, \alpha]$.

It is important to remark that the continuation result of Lemma 1.2 applies to the solutions $\phi_{M}$ and $\phi_{m}$ as well, in the sense that they may be continued as minimum and maximum solutions.

2. Let there exist nonnegative functions $H, Q$, w such that for some $t_{0}>0\left(t_{0}=+\infty\right.$ not excluded $):$

$$
\begin{aligned}
& |h(t)| \leqq H(t), \quad|q(t)| \leqq Q(t), \quad|f(t, x)| \leqq w(t,|x|) \\
& \left(0 \leqq t<t_{0},|x|<\infty\right) .
\end{aligned}
$$

The scalar "comparison" integral equation associated with system (1):

$$
r(t)=H(t)+\int_{0}^{t} Q(t-\tau) w(\tau, r(\tau)) d \tau \quad\left(0 \leqq t<t_{0}\right)
$$

plays an important role in what follows. 
Theorem 2.1. Let $(2,3)$ be satisfied. Let $H$ be continuous on $0 \leqq t<t_{0}$; let $Q \in L$ on every finite subinterval of $\left[0, t_{0}\right)$. Let $w$ be continuous in $(t, r)$ for $0 \leqq t<t_{0}, 0 \leqq r<\infty$. Let $w(t, r)$ be nondecreasing in $r$ for each fixed $t$. For some $b \leqq t_{0}$ let $r_{M}$ be the maximum solution of (8) on $0 \leqq t<b$. Then if $\phi$ is a solution of (1), $\phi$ can be continued to the right as far as $r_{M}$ exists and

$$
|\phi(t)| \leqq r_{M}(t) \quad(0 \leqq t<b) .
$$

In particular if $r_{M}(t)$ exists for $0 \leqq t<\infty$, one has global existence for solutions of (1). If in this case $r_{M}$ is bounded, all solutions of (1) have a bounded norm.

We remark that our approach including the above comparison result and the applications of $\$ 3$ (except for the uniqueness theorem which is similar to ours) is quite different from that of Satō [10]. With more stringent continuity hypotheses the case $n=1$ is considered and the comparison theorem in [10] is in fact an easy generalization of the first part of Lemma 2.1 below, while here the comparison is between the solutions of the system (1) and the maximum solution of the single equation (8). The notion of maximum (but not minimum) solution is introduced in [10], using the comparison theorem proved there, with the result that under our assumptions of Lemma 1.3 above the maximum solution as defined in [10] need not exist. The author in [10] then compares his maximum solution of the single equation corresponding to (1) with solutions of a related integral inequality. This leads to theorems quite different from ours; these do not lend themselves to the type of applications considered in the latter part of $\$ 3$.

The proof of Theorem 2.1 depends in part on a result which also has an analogue in differential equations; compare [6, p. 83].

Lemma 2.1. Let $H, Q$, w satisfy the hypothesis of Theorem 2.1, and let $r_{M}$ be the maximum solution of (8) on $0 \leqq t<b \leqq t_{0}$. For each $\epsilon \geqq 0$ the integral equation

$$
r(t)=H(t)+\epsilon+\int_{0}^{t} Q(t-\tau) w(\tau, r(\tau)) d \tau
$$

has (by Lemma 1.1) at least one solution (which need not exist on the entire interval $0 \leqq t<b)$. Choose one such solution for each $\epsilon>0$ and denote it and its continuation (as far as it exists-Lemma 1.2) by $r(t, \epsilon)$. If $\epsilon_{1}>\epsilon_{2} \geqq 0$ then $r\left(t, \epsilon_{1}\right)>r\left(t, \epsilon_{2}\right)$ for as long as they exist. Let $0<\beta<b$. Then there exists an $\epsilon_{0}>0$ such that each solution $r(t, \epsilon), 0 \leqq \epsilon<\epsilon_{0}$, exists on $0 \leqq t \leqq \beta$, and 


$$
\lim _{\epsilon \rightarrow 0^{+}} r(t, \epsilon)=r_{M}(t)
$$

uniformly with respect to $t$.

To prove the theorem let $\phi$ be any continuous solution of (1) on some interval $0 \leqq t<c \leqq \beta$ and let $m(t)=|\phi(t)|$. By Lemma 2.1 to prove part of the conclusion it suffices to show that $m(t) \leqq r(t, \boldsymbol{\epsilon})$, $0 \leqq t<c, \epsilon$ sufficiently small. It is then shown that the assumption of existence of values of $t, 0 \leqq t<c$, such that $m(t)>r(t, \epsilon)$ leads to a contradiction. For, if $\sigma=\operatorname{glb}[t ; m(t)>r(t, \epsilon)]$, then by continuity $m(\sigma)=r(\sigma, \boldsymbol{\epsilon})$ and $m(t) \leqq r(t, \boldsymbol{\epsilon}), 0 \leqq t \leqq \sigma$. But then from $(1,7)$ and the monotonicity of $w$ it follows after several estimates that $m(\sigma)$ $<r(\sigma, \epsilon)$ which contradicts the definition of $\sigma$ and proves (9) for $0 \leqq t<c$. Application of Lemmas 2.1 and 1.2 permits continuation of $\phi$ to $[0, b)$ with $m(t)<r(t, \boldsymbol{\epsilon}), 0 \leqq t<b, \epsilon$ sufficiently small. This completes a sketch of the proof of Theorem 2.1.

To obtain a lower bound for solutions of (1) requires the following change in assumptions. In place of $H$ in (7) assume the existence of a function $H^{*}$ such that $0 \leqq H^{*}(t) \leqq|h(t)|, 0 \leqq t<t_{0}$. The comparison equation which is used in place of (8) is

$$
\rho(t)=H^{*}(t)-\int_{0}^{t} Q(t-\tau) w(\tau, \rho(\tau)) d \tau .
$$

THEOREM 2.2. Let the hypothesis of Theorem 2.1 be satisfied with $H^{*}$ in place of $H$ and assume that $w$ is nonincreasing in $\rho$ for each fixed $t$, $0 \leqq t<t_{0}$. For some $b \leqq t_{0}$ let $\rho_{m}(t)$ be the minimum solution of (11) on $0 \leqq t<b$. Then whenever $\rho_{m}(t) \geqq 0$, and a solution $\phi$ of (1) exists, $|\phi(t)|$ $\geqq \rho_{m}(t)$.

Obvious changes in Lemma 2.1 are required as well.

3. The methods of $\S 2$ give the following general uniqueness result which includes the uniqueness statement of Lemma 1.1.

THEOREM 3.1. Let $W$ be a continuous nonnegative function on $0 \leqq t<t_{0}$, $r \geqq 0$, and let $W$ be nondecreasing in $r$ for each fixed $t ; W(t, 0)=0$. For each $0<b \leqq t_{0}$ let $\rho(t)=0$ be the only solution of the integral equation

$$
r(t)=\int_{0}^{t}|q(t-\tau)| W(\tau, r(\tau)) d \tau \quad(0 \leqq t<b) .
$$

Let $(2,3)$ be satisfied and assume that

$$
\left|f\left(t, x_{1}\right)-f\left(t, x_{2}\right)\right| \leqq W\left(t,\left|x_{1}-x_{2}\right|\right)\left(0 \leqq t<b ;\left|x_{1}\right|,\left|x_{2}\right|<\infty\right) .
$$

Then there exists at most one solution of (1) on $0 \leqq t<b$. 
It is well known that for differential equations uniqueness itself does not imply convergence of the Picard successive approximations, see [4, pp. 53-54]. However, the hypotheses of a general uniqueness theorem, which are similar to those in Theorem 3.1 and include the monotone requirement on $W$, are sufficient to insure this. It is an open question even for differential equations whether the monotonicity requirement can be removed. Another application of our method yields a criterion for the convergence of successive approximations.

TheOREM 3.2. Let the hypothesis of Theorem 3.1 be satisfied and let $\alpha$ be as in Lemma 1.1. Then the successive approximations

$$
\phi_{0}(t)=0, \quad \phi_{k+1}(t)=h(t)+\int_{0}^{t} q(t-\tau) f\left(\tau, \phi_{k}(\tau)\right) d \tau
$$

converge to the (unique) continuous solution $\phi(t)$ of (1), uniformly on $[0, \alpha]$.

Without the uniqueness assumption of Theorem 3.1, the successive approximations can be shown to have a uniformly convergent subsequence which, however, need not converge to a solution.

As a direct application of Theorem 2.1 we have the following more explicit results. Suppose that $|h(t)|,|q(t)|$ are uniformly bounded on $0 \leqq t<\infty$. Then we may choose $H, Q$ in (7) as

$$
H(t), Q(t)=K \quad(0 \leqq t<\infty),
$$

for some constant $K>0$. The comparison equation (8) is now equivalent to the scalar differential equation

$$
r^{\prime}=K w(t, r) \quad\left(r(0)=K,^{\prime}=d / d t\right) .
$$

Clearly every existence and boundedness theorem for (13) may be translated, via Theorem 2.1, into a similar result for solutions of (1). We refer to Brauer [3] for several such theorems regarding (13). For the application of Theorem 2.1 to integral equations the monotone requirement for $w$ must be retained. A typical example is the following analogue of Wintner's result [9] for differential equations.

Theorem 3.3. Let $(2,3,7,12)$ be satisfied with $t_{0}=+\infty$. Let w $(t, r)$ $=\lambda(t) \theta(r) \quad(0 \leqq t<\infty, 0 \leqq r<\infty)$ and let $\theta$ be nondecreasing. If $\int_{K}^{\infty} d u / \theta(u)=+\infty$ then all continuous solutions of (1) exist on $[0, \infty)$. If further $\int_{0}^{\infty} \lambda(s) d s<\infty$ all continuous solutions of (1) are bounded on $[0, \infty)$.

Other cases of interest may be cited by means of different choices of $H, Q$, in (7). For example in a nonlinear problem in nuclear reactor 
theory (see $[7 ; 8]$ where the linear problem is considered) $h, q$ in (1) are such that $H, Q$ may be taken as

$$
H(t)=K_{1} t+K_{2}, \quad Q(t)=K_{1} t, \quad K_{1}, K_{2}>0, \quad 0 \leqq t<\infty .
$$

This choice makes (8) equivalent to the second order problem

$$
r^{\prime \prime}=K_{1} w(t, r) \quad\left(r(0)=K_{2}, r^{\prime}(0)=K_{1} ; 0 \leqq t<\infty\right)
$$

which is more involved than (13). It can be shown that if $w(t, r)$ $=L(r), 0 \leqq t<\infty$ and if

$$
\int_{K_{2}}^{\infty}\left(K_{1}+\int_{K_{2}}^{8} L(\sigma) d \sigma\right)^{-1 / 2} d s=+\infty
$$

then all solutions of (15) exist for $0 \leqq t<\infty$. Thus in this case if $L$ is nondecreasing and (14) holds, Theorem 2.1 gives global existence for solutions of (1).

Finally, it is hoped that by this method we may obtain other results for integral equations, e.g. the case when (8) is not equivalent to a differential equation, approach to limits and stability of solutions by exploiting the fact that the right hand side of (8) defines a positive operator on the space of continuous functions.

\section{BIBLIOGRAPHY}

1. H. A. Antosiewicz, An inequality for approximate solutions of ordinary differential equations, Math. Z. 78 (1962), 44-52.

2. F. Brauer, Global behavior of solutions of ordinary differential equations, J. Math. Anal. Appl. 2 (1961), 145-158.

3. - Bounds for solutions of ordinary differential equations, Proc. Amer. Math. Soc. (to appear).

4. E. A. Coddington and N. Levinson, Theory of ordinary differential equations, New York, McGraw-Hill, 1955.

5. R. Conti, Sulla prolungabilita delle soluzioni di un sistema di equazioni differenziali ordinarie, Boll. Un. Mat. Ital. 11 (1956), 510-514.

6. E. Kamke, Differentialgleichungen Reeler Funktionen, Leipzig, 1956.

7. J. J. Levin and J. A. Nohel, On a system of integrodifferential equations occurring in reactor dynamics, J. Math. Mech. 9 (1960), 347-368.

8. - On a system of integrodifferential equations occurring in reactor dynamics. II, Arch. Rational Mech. Anal. (1962) (to appear).

9. A. Wintner, Ordinary differential equations and Laplace transforms (appendix), Amer. J. Math. 79 (1957), 265-294.

10. T. Satō, Sur l'équation intêgrale non linéaire de Volterra, Composito Math. 11 (1953), 271-290.

UNIVERSITY OF WISCONSIN 